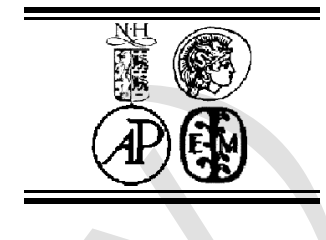

\title{
Robust spatially resolved pressure measurements using MRI with novel buoyant-advection free preparations of stable microbubbles in polysaccharide gels
}

\author{
Robert H Morris ${ }^{a}$, Martin Bencsik ${ }^{a,}{ }^{*}$, Nikolaus Nestle ${ }^{b}$, Petrik Galvosas ${ }^{c}$, David Fairhurst ${ }^{a}$, \\ Anil Vangala ${ }^{\mathrm{d}}$, Yvonne Perrie ${ }^{\mathrm{e}}$, Glen McHale ${ }^{\mathrm{a}}$ \\ ${ }^{a}$ Nottingham Trent University, Clifton Lane, Nottingham, NG11 8NS, UK \\ ${ }^{b}$ BASF Aktiengesellschaft, Ludwigshafen, Germany \\ ${ }^{c}$ Universität Leipzig, Fakultät für Physik und Geowissenschaften, Linnéstraße 5, Leipzig, Germany, \\ ${ }^{d}$ School of Pharmacy and Chemistry, Kingston University, Surrey, KT1 2EE \\ ${ }^{e}$ Medicines Research Unit, School of Life and Health Sciences, Aston University, Birmingham, UK.
}

Elsevier use only: Received date here; revised date here; accepted date here

\begin{abstract}
MRI of fluids containing lipid coated microbubbles has been shown to be an effective tool for measuring the local fluid pressure. However, the intrinsically buoyant nature of these microbubbles precludes lengthy measurements due to their vertical migration under gravity and pressure-induced coalescence. A novel preparation is presented which is shown to minimize both these effects for at least 25 minutes. By using a $2 \%$ polysaccharide gel base with a small concentration of glycerol and 1,2-Distearoyl-sn-Glycero-3-Phosphocholine coated gas microbubbles, MR measurements are made for pressures between 0.95 and 1.44 bar. The signal drifts due to migration and amalgamation are shown to be minimized for such an experiment whilst yielding very high NMR sensitivities up to $38 \%$ signal change per bar.
\end{abstract}

(C) 2002 Elsevier Science. All rights reserved.

Keywords: Gas filled lipid coated microbubbles; Pressure; Polysaccharide; MRI; Diffusion; Viscosity; Contrast agent

\footnotetext{
* Corresponding author. Tel: +44 1158488057 Fax: +44 1158486636

E-mail address:martin.bencsik@ntu.ac.uk
} 


\section{Introduction}

Until recently, fluid pressure measurements were made exclusively with discrete electrical or mechanical sensors at the surface of samples. If a suitable fluid is used [1], MRI can non-invasively produce 3-D images of fluid pressure. Applications as diverse as diagnostic medical imaging, fundamental research for chemical engineering and enhanced oil recovery are possible with such a technique, which could revolutionise the understanding of many processes where spatial variations of fluid pressure exist, such as for the case of a flowing viscous fluid. Since 1996 two methods both utilising MRI have been demonstrated: for samples which have low permeability, compressible fluids such as gasses can be used to yield pressure sensitivity through density measurement [1] although this is not suitable for samples such as sandstones; an alternative method takes advantage of the susceptibility difference between lipid coated, micron sized gas bubbles (microbubbles) and a suspending liquid medium. When subjected to a magnetic field, these microbubbles cause perturbations which are dependent on their radius [2]. A change in pressure causes a change in the size of these compressible microbubbles and therefore a change in the perturbations. Protons undergoing Brownian motion around these microbubbles experience a range of different fields causing signal dephasing which depends on the microbubble size and the distance which they have diffused during the measurements [3].

The microbubble method was originally conceived as a method for in vivo human manometry using MRI [3]. A theoretical study published in 2002 [4] suggested that the amount of gas required for sufficient sensitivity exceeds the lethal dose, unless a sophisticated paramagnetic coating could be used [5]. In 2007 the microbubble preparations were applied to study the pressure of a flowing fluid in sandstone-like samples [6].

In the previous works it has been shown that the preparations present instabilities over time which cause drifts in the NMR signal. Lipid coated microbubbles are prone to damage as a result of the application of pressure, owing to the fragile properties of their membranes and the propensity of the gas to dissolve into the surrounding fluid. This effect coupled with their intrinsic buoyancy must be counteracted to make meaningful measurements over time.

Presented here is a novel preparation which succeeds in minimising these effects whilst maintaining a high sensitivity to pressure changes using MRI. Rheometry, diffusion NMR, time lapse photography and ultra-fast MRI are used to validate the properties of the preparation.

\section{Theory}

\subsection{Origin of the NMR sensitivity}

The susceptibility difference between two media in a static magnetic field causes local polarising field perturbations. The susceptibility difference between water and air is in the order of $10^{-6}$ [7] causing microbubbles to produce perturbations extending an appreciable distance from their surfaces. Water molecules which are diffusing in such a field will be subjected to a range of field strengths different to that in bulk fluid. The relative perturbations of the longitudinal component of the polarising field surrounding a sphere with a magnetic susceptibility difference of $\Delta \chi$ to its surroundings are given by [2]:

$$
\frac{\Delta B_{z}(r, \theta)}{B_{o}}=\frac{4 \pi}{3} \Delta \chi\left(\frac{R}{r}\right)^{3}\left(3 \cos ^{2} \theta-1\right), \quad \text { Eq. } 1
$$

where $R$ is the radius of the sphere and $r$ is the radial distance from its centre at angle $\theta$ from the direction of the static magnetic field of strength $B_{0}$.

Because the length scale of these perturbations is dependent on the microbubble radius, a change in bubble size will result in a change in the distribution of the field strengths sampled by a water molecule diffusing in the liquid, resulting in a change of signal intensity. As the microbubbles are compressible, a change in external pressure will cause such a change in radius, yielding MRI pressure contrast. Because the process relies on the diffusion of water molecules around the microbubbles, the distance which they have diffused in a given time affects the sensitivity of the technique: If the protons have not travelled sufficiently far between two comparative acquisitions, no signal change will be seen; if they travel too far, they do not encounter sufficient perturbations either. It has been shown [4] that dephasing is maximised when molecules are allowed to diffuse a specific distance in terms of the length scale of these perturbations, and is partially dependant on the NMR sequence that is used.

\subsection{Bubble rise velocity}

The most important factor affecting the stability of the measurements over time is the ability of the suspending medium to prevent buoyant advection of the microbubbles. The expected rise velocity for a microbubble can be determined by equating the two opposing forces which act on it; the buoyant force, 


$$
F_{B}=\frac{4}{3} \pi R^{3} \Delta \rho g
$$

and the viscous drag on a sphere, which is given by Stokes' law for the case of low Reynolds number,

$$
F_{D}=6 \pi \eta R \mathrm{v}
$$

where $\mathrm{R}$ is the radius of a sphere moving with velocity $\mathrm{v}$ through a fluid with viscosity $\eta, g$ is the acceleration due to gravity and $\Delta \rho$ is the difference in density between the gas and the liquid.

Because the lipids in the membranes of the microbubbles are in the gel state, they have a very low mobility, and they are not likely to cause additional slip at the interface between the bubble and the liquid.

Several models are available to describe the properties of non-Newtonian fluids. In the case of our study, the viscosity of pseudo-plastic fluids is described by the power law model [8],

$$
\eta=\mathrm{k}_{2} \dot{\gamma}^{n-1}, \quad \text { Eq. } 4
$$

which provides excellent correlation with the experimental data. In the previous expression $\dot{\gamma}$ is the shear rate, whilst the consistency, $\mathrm{k}_{2}$, and the power law index, $\mathrm{n}$, are two parameters that fully describe the rheological properties of the fluid.

For a particle moving through such a power-law fluid, the particle Reynolds number is given by [9]:

$$
\operatorname{Re}_{p}=\frac{\Delta \rho \mathrm{v}^{2-n}(2 R)^{n}}{\mathrm{k}_{2}} \cdot \quad \text { Eq. } 5
$$

To estimate the maximum possible Reynolds number in the experimental system, the following 'worst case' parameters are assumed: $\Delta \rho=1.1 \mathrm{~kg} \cdot \mathrm{m}^{3}, v=5 \times 10^{-7} \mathrm{~m} \cdot \mathrm{s}^{-1}, R$ $=20 \mu \mathrm{m}, n=0.3$ and $k_{2}=10 \mathrm{~Pa} \cdot \mathrm{s}^{0.3}$. This gives a Reynolds number less than $5 \times 10^{-12}$ which is considerably lower than values associated with a turbulent regime, corresponding to $R e_{p}>1$.

According to Williams [10] the shear force caused by a sphere moving with low Reynolds number can be approximated by

$$
\dot{\gamma}=\frac{\mathrm{v}}{2 R} .
$$

By equating the buoyant and drag forces, and using the 'worst case' parameters and models mentioned previously, the terminal velocity of a sphere with a given radius can be expressed in terms of the physical properties of a fluid:

$$
\mathrm{v}=\left[\frac{2^{n} R^{n+1} \Delta \rho g}{9 k_{2}}\right]^{\frac{1}{n}}
$$

For a $5 \% \mathrm{v} / \mathrm{v}$ suspension, the highest concentration explored in this work, the microbubbles are separated by more than four times their own diameter, and in consequence hydrodynamic interactions between neighbouring microbubbles can be neglected.

\subsection{MRI sequence}

In all MRI work presented here, the RARE sequence [11] is used with the RARE factor set to the number of lines in the images, allowing an image to be collected from a single excitation pulse as in EPI.

To ensure that the sensitivity of the experiment is maximised, the effective echo time, $\mathrm{T}_{\mathrm{E}}{ }^{\text {eff }}$ must be optimised with respect to the $\mathrm{T}_{2}{ }^{\text {eff }}$ of the sample. The optimum setting required to yield maximum sensitivity to small changes in $\mathrm{T}_{2}{ }^{\text {eff }}$ is determined both semi-analytically and using MonteCarlo simulation in MATLAB (Mathworks, MA, USA).

For a homogeneous sample defined as in Figure 1, by integrating the NMR signal along the $y$-axis and assuming that the exponential decay during the read gradients can be neglected as the encoding time is sufficiently short, the signal intensity for a modulus MRI image is well approximated by:

$$
A\left(T_{2}^{\text {eff }}, T_{E}^{\text {eff }}\right)=\int_{o}^{2 T_{E}^{e f f}}\left|2 \frac{\sin \left(\gamma G_{y} y_{o}\left(t-T_{E}^{e f f}\right)\right)}{\gamma G_{y}\left(t-T_{E}^{e f f}\right)}\right| e^{\left(-t / T_{2}^{e f f}\right)} d t .
$$

where $\gamma$ is the proton gyromagnetic ratio (not related to $\dot{\gamma}$ used in the rheology section), whilst $\mathrm{G}_{\mathrm{y}}$ is the strength of the phase encoding imaging field gradient, $t$ is the time and $\mathrm{y}_{\mathrm{o}}$ is the extent of the sample along the $\mathrm{y}$-axis.

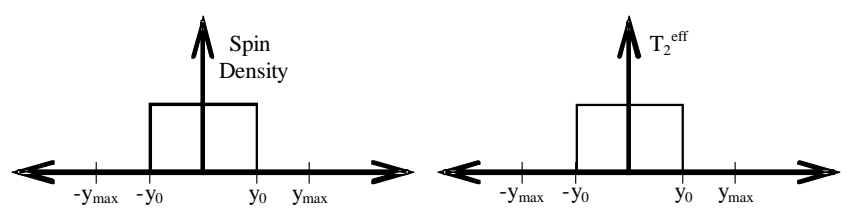

Figure 1: Simple homogeneous sample profiles used to estimate optimum echo time. 
For a small change in $\mathrm{T}_{2}$ eff , the greatest change in signal intensity is given (see Appendix) by

$$
T_{E}^{e f f}=2 T_{2}^{e f f}
$$

Although quantitatively correct, for practical MRI purposes the qualitative deterioration of an image that would be acquired with this set up needs to be assessed. This is achieved using Monte Carlo simulation, in which an idealised circular homogeneous image has its corresponding train of spin echoes multiplied by an exponential decay for a range of different $\mathrm{T}_{2}{ }^{\text {eff }}$ values before thermal noise is added. The mean amplitude of the resulting MR image is then calculated in terms of $\mathrm{T}_{\mathrm{E}}{ }^{\text {eff }}$.

Visual inspection of the reconstructed images allows qualitative estimation of the image artefacts, particularly those due to the increasing attenuation of the centre of kspace. Quantitation of the SNR (mean signal divided by the standard deviation of noise) in the set of generated images also permits verification of the result in Eq. 8 .

\section{Materials}

In order to minimise the extent to which microbubbles dissolve in the surrounding medium, they are coated with a lipid shell. These lipid coated microbubbles are commonly used as a contrast agent in ultrasound as they greatly enhance the scattering of the incident wave, thereby improving contrast [12].

There are numerous methods for producing these microbubbles ranging from simple sonication [13] methods to very complex freeze drying procedures [14]. The method used for this work is detailed by Vangala et al. [15] and uses high shear mixing to produce polydisperse microbubbles. The chosen lipid powder is hydrated with water, forming laminar sheets. The mixture is then subjected to high shear mixing at atmospheric pressure using a homogeniser, spontaneously introducing gas bubbles. The lipids are in their lowest energy state when their hydrophobic chains are inside the gas bubbles whilst their hydrophilic head groups are in the surrounding medium. As a consequence of this, the lipids form a membrane at the gas liquid interface of the microbubbles. This method allows rapid and efficient production of gas filled lipid coated microbubbles $1-20 \mu \mathrm{m}$ in size. The lipid used for the following work is 1,2-Distearoyl-sn-Glycero3-Phosphocholine (DSPC, C18:0, MR: 790.16) as this has been shown to yield superior stability in comparison to shorter chain lipids [13].
To prevent buoyant advection of lipid coated microbubbles through the suspending medium, a highly viscous preparation is necessary. However, according to the Stokes-Einstein relationship, higher viscosity results in lower diffusion for Newtonian fluids. Since the diffusion of water molecules around the lipid coated microbubbles is critical for the MRI contrast, simply increasing the viscosity may greatly reduce the sensitivity. However, nonNewtonian fluids are not governed by the Stokes-Einstein relationship. Polysaccharide gels are an example of a group of fluids which allow increase in viscosity with minimal effect on the diffusion of water within their structure [16]. We present results that demonstrate that this is the case for gellan gum, which is the polysaccharide gel used in these experiments, and justify its use as a suitable suspending medium for this contrast agent.

gellan gum in aqueous solution forms an entangled polymer network at relatively low concentrations (typically less than $5 \%$ ), rapidly increasing the bulk viscosity of the fluid [17]. Because the polymer chains are very long, the majority of water molecules in the solution are free to diffuse unhindered resulting in minimal reduction in the diffusion coefficient. Unfortunately, polysaccharide gels have a high propensity to support bacterial growth which can change many properties of the gel including the viscosity. In order to minimise the bacterial growth, Sodium Hypochlorite is used in a concentration of $0.02 \%$ $\mathrm{v} / \mathrm{v}$. Lipid coated microbubbles have been tested in this solution to ensure that it does not affect their stability (which would not be expected as they are not electrically charged).

The gellan gum is supplied in powdered form, and for these experiments CPKelco's Kelcogel AFT (CPKelco, USA) is used. This is an industrial grade gum with a particle size of $355 \mu \mathrm{m}$ [18] which must be rehydrated with water to produce a gel. To prevent inclusion of gas bubbles within the gel, which could lead to spurious measurements, it is hydrated under vacuum.

Prior to the suspension of microbubbles in the gel, they are blended with an equal concentration of glycerol. The reason this increases the stability of the microbubble is unknown at this time, but it has been seen to somewhat reduce the drift in signal intensity.

\section{Experimental}

The properties of the polysaccharide gel are thoroughly analysed before it is used as the base of the contrast agent. The fluid should reduce the buoyant advection of the microbubbles with minimal restriction to the self-diffusion of water within its structure which in turn should not affect the lipid coated micro-bubbles. 
The viscosity of the gel is measured at various concentrations for a range of shear rates using a TA Instruments (Delaware, USA) $\mathrm{CSL}^{2}$ Rheometer. The instrument is calibrated using PolyDiMethylSiloxane (PDMS) oils which are available in a wide range of viscosities over which they are highly Newtonian. The instrument is used with a 'Mooney-Ewart' geometry [19] tool manufactured from acrylic. The viscosity is measured for increasing and decreasing shear rates allowing the rheological properties of the gel to be studied for a static and flowing fluid.

To verify that the prediction of the bubble rise velocity is representative of the practical situation, turbidity measurements of two samples, methyl cellulose and gellan gum prepared in equal concentration is performed. The opacity of the microbubbles is sufficient that time lapse photography can be used. The samples are photographed simultaneously every minute using an 8-bit black and white DSA Mk 10 camera (Krüss, Hamburg, Germany). In order to prevent clipping, the contrast of the images is adjusted by varying the light intensity until an image of the gels without microbubbles gives a grey value of 250 , allowing the smallest possible microbubbles to be observed. To facilitate simultaneous display of the collected data, the intensity across the width of each sample is averaged, providing vertical profiles each minute. These are plotted and the gradients of similar intensities calculated to determine the rise velocity of various microbubble sizes. These are then compared to the theoretical estimates in an attempt to validate them.

The self-diffusion of water contained in the fluid is measured using a $400 \mathrm{MHz}$ pulsed field gradient spectrometer at University of Leipzig. The magnet is a wide bore 9.4T superconducting type (Bruker, Germany) and is used with a MARAN ULTRA console (Resonance Instruments, GB) with a Direct Binary Coded Current Source [20] for gradient pulse generation, whilst the instrument is triggered at a constant mains voltage phase to avoid hum artefacts. Using a stimulated gradient echo sequence with compensation for a shift in echo position caused by imperfect gradient pulses [20], a sample volume of $0.5 \mathrm{ml}$ is tested in the actively shielded anti-helmholtz type probe at a range of temperatures for distilled water and gellan gum. These experiments allow rapid assessment of the stability of the preparation and its sensitivity to minor changes in temperature.

Once the fluid properties have been assessed, it is prepared for use as a contrast agent to pressure variation. The dry powder is dissolved in distilled water to a concentration of $2 \% \mathrm{w} / \mathrm{v}$ whilst stirring vigorously for 8 hours under vacuum (450 torr) until a gas free homogeneous preparation is achieved. Lipid coated microbubbles are then blended with an equal concentration of glycerol before mixing with the gellan gum to a concentration of $1.25 \% \mathrm{v} / \mathrm{v}$. Having achieved a homogeneous preparation, the fluid can be used directly as an MRI contrast agent to pressure.

The MRI experiments are performed using a 2.35T Biospec small animal scanner (Bruker, Germany). As the fluid is static, ultra fast imaging can be used without concern for flow artefacts.

Two MRI experiments are conducted: The first is to test for the presence of microbubble advection. A single large volume of fluid is tested in a cylindrical acrylic cell $(42 \mathrm{~mm}$ id x 70mm) with Swagelok (Swagelok, USA) connectors at the inlet and outlet. The pressure of this sample is then varied using a remotely connected syringe pump whilst the RARE sequence is run. The signal intensity at several pressures is divided by the average signal intensity over all pressures. If the microbubbles experience advection during the experiment, a vertical gradient will develop in time. This is tested by plotting profiles from a single line across the centre of the sample horizontally and vertically.

The second experiment tests the stability of the MR sensitivity to pressure changes. Using a similar experimental setup, the fluid pressure is varied whilst it is monitored and recorded using a traditional piezoelectric pressure sensor. The average signal over the sample volume is plotted alongside the recorded pressure to test for correlation between external pressure and the signal intensity. A control sample containing just gellan gum is also tested to ensure that sensitivity is indeed coming from the presence of lipid coated microbubbles and not from trapped gas or other unintentional contrast mechanisms.

\section{Results}

\subsection{Viscosity Measurements}

The viscosity of the gel is measured for three samples shown on Figure 2. The plots show the variations in viscosity for a range of shear rates. The consistency and power law index are calculated from a log-log plot (see Figure 2). The viscosity is also measured for $2 \%$ methyl cellulose (which was used in two previous studies $[3 ; 6]$ ) for comparison to the properties of the gellan gum (see Figure 3). For shear rates below $12 \mathrm{~s}^{-1}$ the gellan gum's viscosity is far higher than the methyl cellulose, above this value, viscosities are similar within 0.25 Pas. 


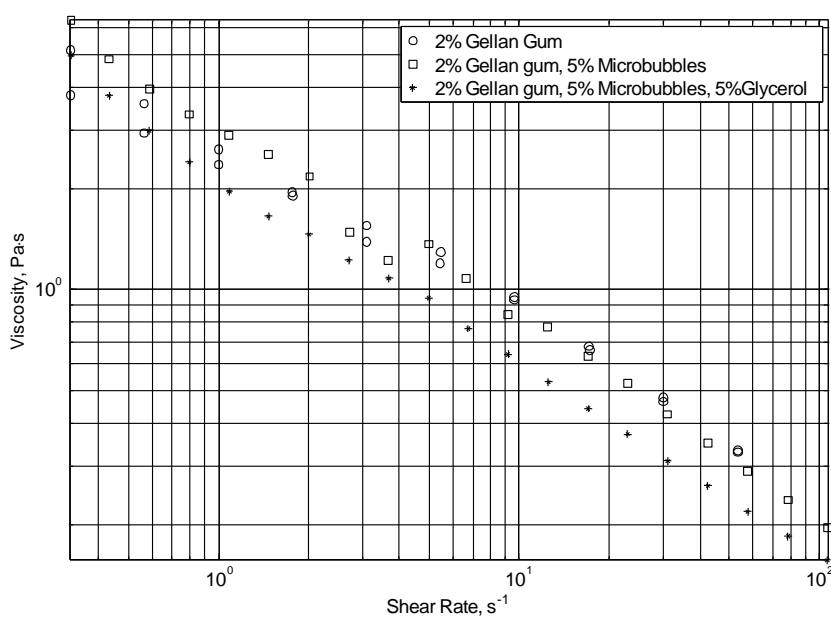

Figure 2: Log-log plot of viscosity against shear rate for the three samples. The addition of glycerol and microbubbles has little effect on the viscosity of the fluid, even at concentrations as high $5 \%$. At low shear the viscosity of the fluids are very high with a value greater than 6 Pas. The plot demonstrates near ideal power law behavior and allows determination of the rheological coefficients, consistency $\left(\mathrm{k}_{2}\right)$ and power law index (n).

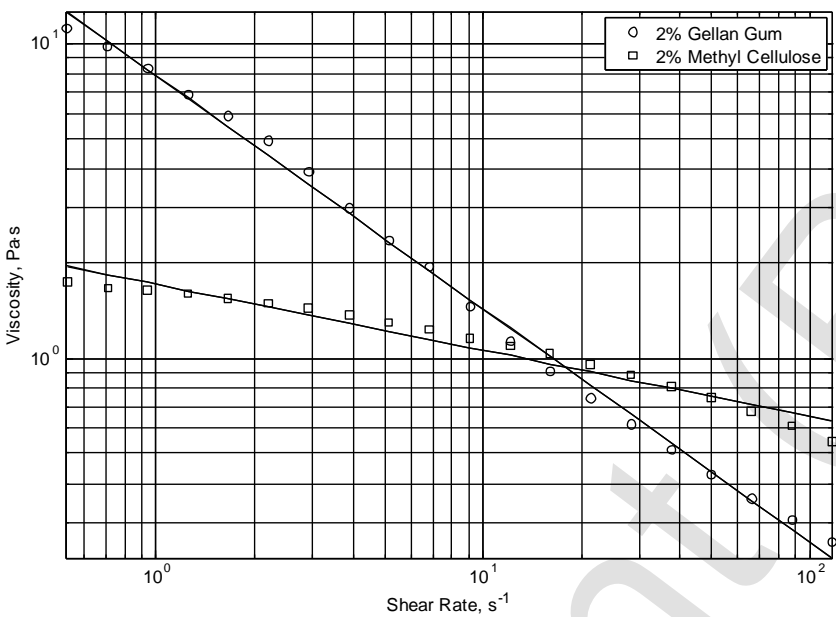

Figure 3: Log-log plot of viscosity against shear rate for gellan gum and methyl cellulose both at $2 \% \mathrm{w} / \mathrm{v}$ demonstrating near ideal behavior and allowing determination of consistency $\left(\mathrm{k}_{2}\right)$ and power law index $(\mathrm{n})$.

\subsection{Microbubble advection measurements}

The theoretical velocities predicted for a range of microbubble sizes determined using Eq. 6 and rheology data in Figure 3 are plotted in Figure 4. The expected velocity for a microbubble in methyl cellulose is predicted to be three orders of magnitude faster than a similarly sized microbubble in gellan gum (e.g. for $13 \mu \mathrm{m}$ bubbles, velocities are $8 \times 10^{-8} \mathrm{~m} \cdot \mathrm{s}^{-1}$ and $2 \times 10^{-13} \mathrm{~m} \cdot \mathrm{s}^{-1}$ for methyl cellulose and gellan gum respectively.)

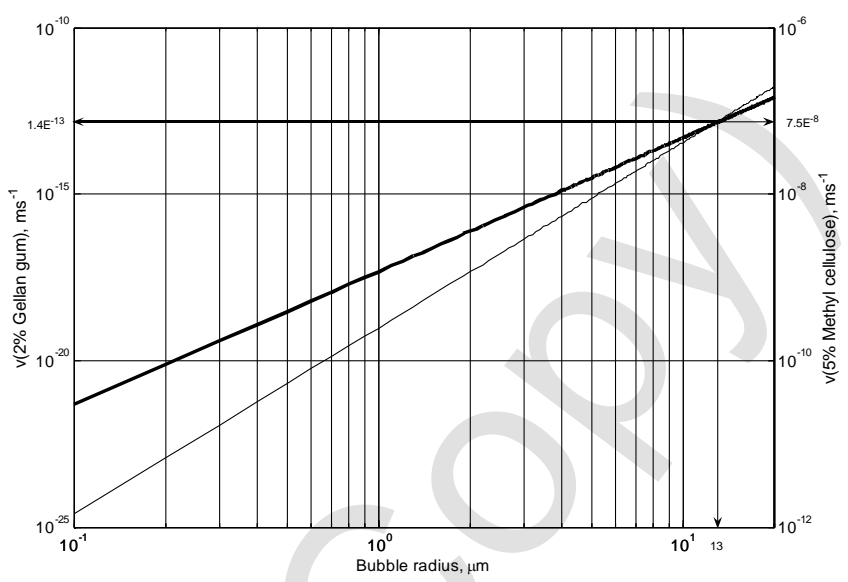

Figure 4: Log-log plot of predicted rise velocity (v) for a given microbubble radius based on extrapolated rheology data (the lowest shear rate value measured in Fig 3 corresponds on this graph to bubble radii of $126 \mu \mathrm{m}$ and $110 \mu \mathrm{m}$ for the Gellan gum and the Methyl cellulose respectively), produced using equations (2-6) for comparison of two preparations.
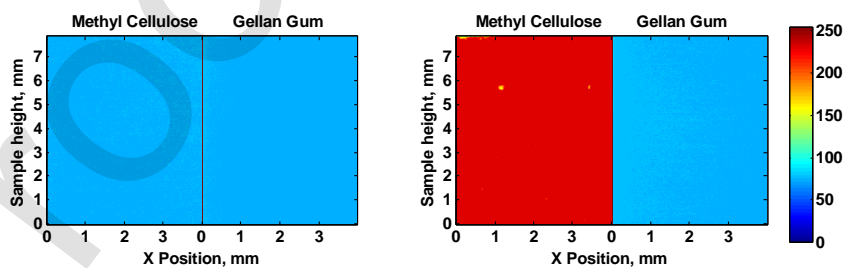

Figure 5: Initial (left) and final (right) image from the time lapse study. The final image is collected 46 hours after the initial image. The left hand side of each image is the methyl cellulose sample whilst the gellan gum is on the rights hand side. The colour codes the intensity of light incident on the camera.
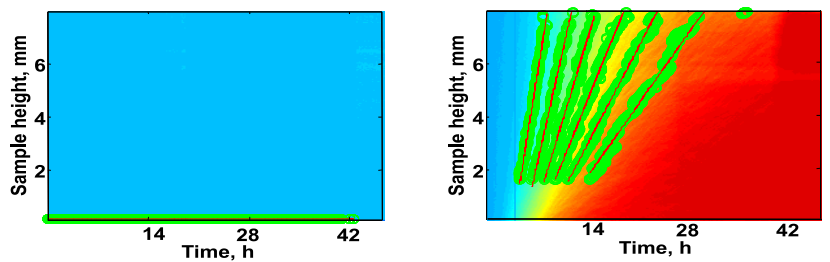

Figure 6: Profile images. The gradients which yield estimates of rise velocity are shown in green on the two images and the gradients calculated are shown as red lines. The colour codes the average intensity of light averaged over the sample width.

Two images from the time lapse photography study are shown in Figure 5 (the original video showing each time lapse image is available as supporting material). In order to present the data in a format which allows rapid assessment of microbubble rise velocity, vertical profiles are taken for each frame from within the two regions bounded by the green outlines. The profiles are then stacked from left to right and shown in Figure 6. By following contours of similar pixel intensity, the velocity of a range of 
microbubbles of similar size may be estimated. These contours are shown in green on Figure 6. The microbubbles are immobile in the gellan gum for the duration of the experiment (46 hours) which corresponds to a velocity less than $1 \times 10^{-12} \mathrm{~m} \cdot \mathrm{s}^{-1}$ whilst velocities ranging from $8 \times 10^{-8} \mathrm{~m} \cdot \mathrm{s}^{-1}$ to $4 \times 10^{-7} \mathrm{~m} \cdot \mathrm{s}^{-1}$ are found for the methyl cellulose.

\subsection{Diffusion Measurements}

The diffusion measurements were conducted over a range of temperatures between $298 \mathrm{~K}$ and $338 \mathrm{~K}$. Five measurements were made for the gellan sample (with a gradient pulse separation time of $3 \mathrm{~ms}$ ), as shown in Figure 7. On the same figure, values for the variations of diffusion coefficients for bulk water as found by Holz et al. [21] are plotted alongside the experimental data to demonstrate the similarity of the diffusion coefficients for gellan gum and bulk water. For the range of temperatures investigated, the diffusion coefficient of water in the gellan gum matrix is similar to that of bulk water within $7 \%$.

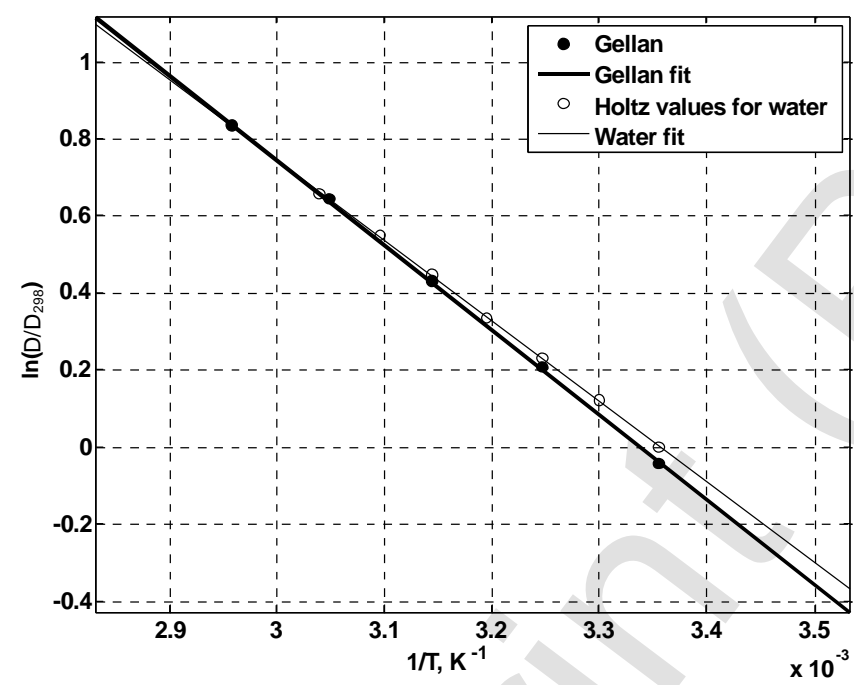

Figure 7: Plot of the natural logarithm of the diffusion coefficient against the reciprocal of temperature, normalized by value of bulk water at room temperature for water and gellan gum. Correlation between samples is high implying that the majority of water in the gel sample is unrestricted by the network.

\subsection{Optimization of signal sensitivity}

The measurement of fluid pressure with MRI offers vast variations in sensitivity, spatial and temporal resolutions due to the large and growing number of MR sequences. It is important to optimize as many parameters as possible to fully enhance the signal sensitivity.

Most often the signal is acquired within a short fraction of the NMR exponential decay, and in this case, the most sensitive measurement of small changes in $T_{2}$ is found when $\mathrm{T}_{\mathrm{E}}=\mathrm{T}_{2}$.

For the case of the static fluids explored in this study, the RARE sequence with single shot planar imaging was found to yield an excellent compromise between spatial and temporal resolutions, whilst the sensitivity to pressure changes could be maximized by setting $T_{E}^{\text {eff }}=2 T_{2}^{\text {eff }}$, as is shown in Figure 8.

The two methods correlate well, although small differences can be seen due to the thermal noise that is not accounted for in the semi-analytical solution.

When the condition $T_{E}^{\text {eff }}=2 T_{2}^{\text {eff }}$ is fulfilled, the centre of $\mathrm{k}$-space suffers from strong attenuation, yielding image artifacts, mostly in the form of DC signal loss (see Figure 9), resulting in spuriously enhanced edges together with reduced intensity of the central volume. By choosing to set $\mathrm{T}_{\mathrm{E}}{ }^{\text {eff }}$ at a value around $1.6 \mathrm{~T}_{2}{ }^{\text {eff }}$, an excellent sensitivity to pressure changes is maintained whilst image artifacts are substantially reduced.

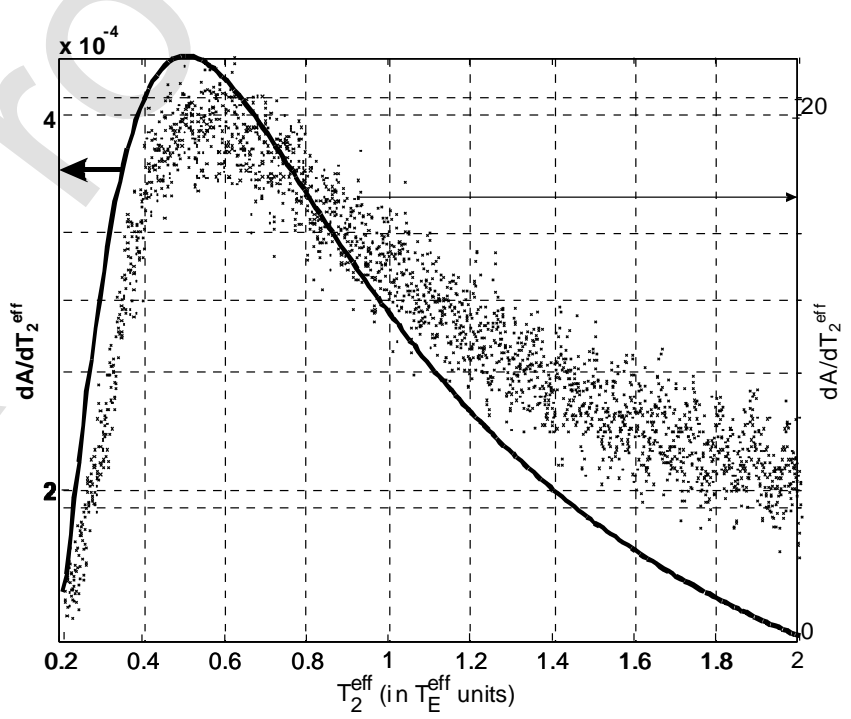

Figure 8: Plot of gradients of semi-analytical (solid line) and Monte Carlo (single points) simulation signals, showing the optimum value for $\mathrm{T}_{\mathrm{E}}{ }^{\text {eff }}$ to measure small changes in the value of $\mathrm{T}_{2}{ }^{\text {eff }}$. A Gaussian convolution (width $=0.5 \mathrm{~T}_{2}{ }^{\text {eff }}$ ) is applied to the MR signal found from the Monte Carlo data prior to obtaining the gradient. Both tests suggest that setting $\mathrm{T}_{\mathrm{E}}$ eff to twice the value of $\mathrm{T}_{2}{ }^{\text {eff }}$ yields optimum sensitivity to a change in $\mathrm{T}_{2}{ }^{\text {eff }}$. 

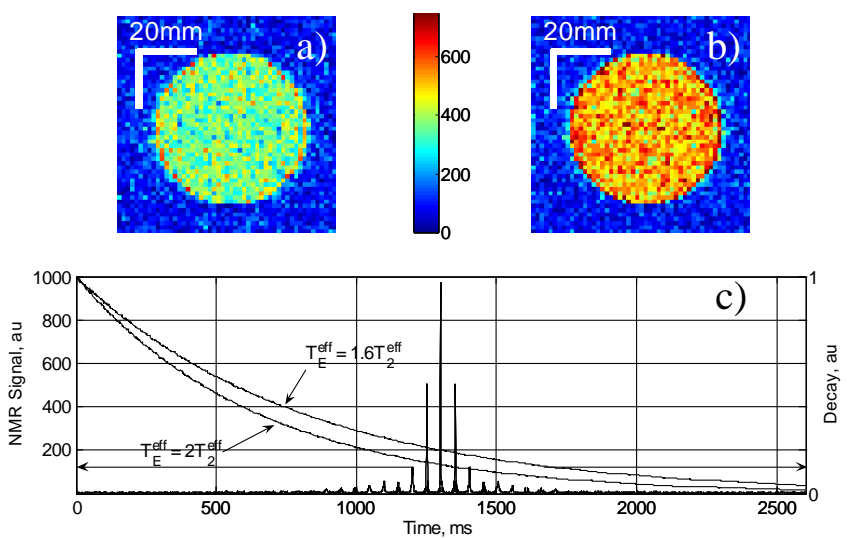

Figure 9: Demonstration of k-space clipping due to length of relaxation. a) image with $\mathrm{T}_{\mathrm{E}}{ }^{\text {eff }}=2 \mathrm{~T}_{2}{ }^{\text {eff; }}$; b) image with $\mathrm{T}_{\mathrm{E}}{ }^{\text {eff }}=1.6 \mathrm{~T}_{2}{ }^{\text {eff }}$; The pixel intensities are in arbitrary units. c) shows the RARE echo train with no relaxation, superimposed with the relaxation curve for the case $\mathrm{T}_{\mathrm{E}}{ }^{\text {eff }}=2 \mathrm{~T}_{2}{ }^{\text {eff }}$, and for the case $\mathrm{T}_{\mathrm{E}}{ }^{\text {eff }}=1.6 \mathrm{~T}_{2}{ }^{\text {eff }}$ (red curves).

\subsection{MRI with contrast agent}

The bulk fluid cell is filled with $2 \%$ gellan gum with distilled water (w/v) and $2.5 \%$ microbubbles and glycerol in equal concentration to test the stability to buoyant advection. The images in Figure 10 show the signal intensity relative to the mean for three pressures. The high homogeneity of the images is indicative of the lack of buoyant advective motion of the microbubbles through the medium. The images are acquired using the RARE sequence with the following imaging parameters: Matrix size $=74 \times 74 ; \mathrm{T}_{\mathrm{E}}=6.737 \mathrm{~ms} ; \mathrm{T}_{\mathrm{E}}{ }^{\text {eff }}=284 \mathrm{~ms}\left(0.6 \mathrm{~T}_{2}{ }^{\text {eff }}\right) ; \mathrm{T}_{\mathrm{R}}=$ $2462 \mathrm{~ms}$ ( $\mathrm{T}_{2}{ }^{\text {eff }}$ of the sample was $424 \mathrm{~ms}$ ). The value of $\mathrm{T}_{\mathrm{E}}{ }^{\text {eff }}$ was not optimised for these experiments as they were intended to demonstrate the reduction in advection whilst a sufficiently high sensitivity was observed. If the value of $\mathrm{T}_{\mathrm{E}}{ }^{\text {eff }}$ had been optimised and set at $636 \mathrm{~ms}$, the sensitivity could have been as high as $96 \%$ signal change per bar.
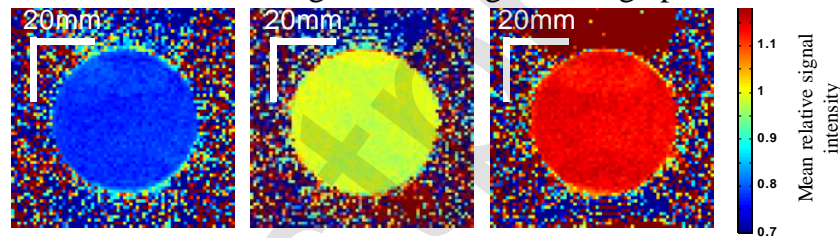

Figure 10: Three MRI images taken at different pressures to demonstrate reduction in migration of microbubbles. The three images are taken at: $0.95,1.3$ and 1.44 bar (left to right respectively).

Single pixel horizontal and vertical profiles from the central row and column of the images in Figure 10 are plotted in Figure 11 to demonstrate the minimization of microbubble advection. The three curves present no measurable gradient which is indicative of no advective processes for the duration of the 25 minute experiment.
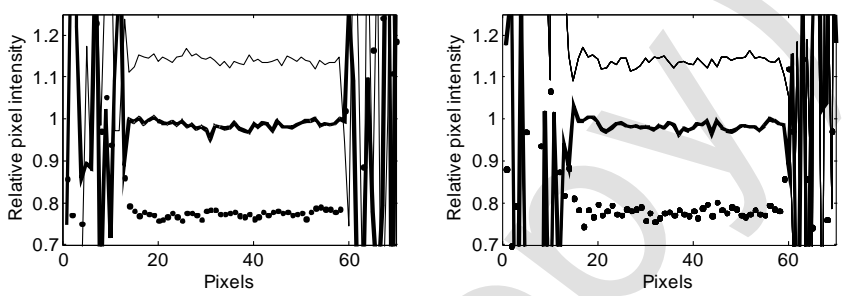

Figure 11: Horizontal (left) and vertical (right) profiles taken across the centre of MRI images in Figure 10. Broken line is for 0.95 bar, thick line for $1.3 \mathrm{bar}$ and thin line for $1.44 \mathrm{bar}$.

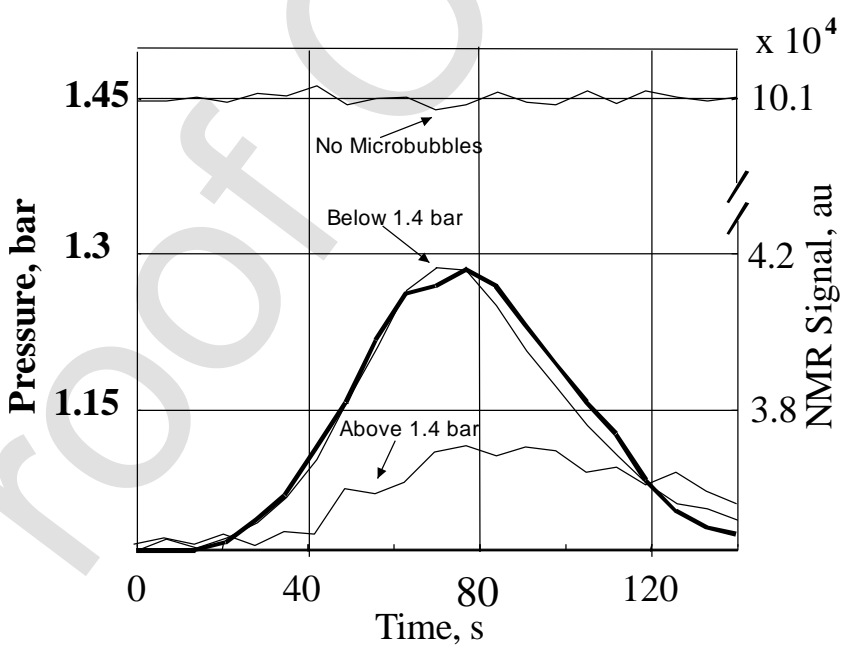

Figure 12: Plot of average signal over sample volume and pressure against time. Thick curve is pressure measured with a piezoelectric pressure sensor whilst thin curves represent the averaged signal intensity. Thin curves are: Control sample containing degassed gellan gum, sample with microbubbles which have not been subjected to pressures in excess of 1.4 bar, and a sample that has experienced pressures above 1.4 bar.

The average signal intensity over the volume is calculated for each scan in time and is plotted alongside the pressure measured using piezoelectric sensors. The correlation is high for a single cycle below 1.4 bar. Once the fluid experiences further cycles at such pressures or if greater pressures are used, the microbubbles rupture or coalesce and reduce the sensitivity from $38 \%$ signal change per bar to less than $10 \%$ signal change per bar (See Figure 12). Data collected after several cycles of pressure, showing this reduction in sensitivity without introducing advection is supplied as a seperate movie in the supporting material.

\section{Discussion}

The three gellan gum samples used for rheological measurements demonstrate similar shear rate - viscosity profiles despite inclusion of microbubbles and glycerol. The viscosity of the gellan gum is considerably higher than 
that of the methyl cellulose in equal concentration provided that the shear forces experienced are below $12 \mathrm{~s}^{-1}$, which would correspond to a flow rate of $2.9 \mathrm{mms}^{-1}$ in a $10 \mu \mathrm{m}$ pipe.

For the MRI experiments conducted in this paper, the fluid is at rest. In consequence, the only shear forces exerted on the fluid come from microbubble advection. For optimum MRI stability, and to prevent measurable drifts, the rise velocity should be sufficiently small to prevent microbubbles moving out of a voxel over the experimental duration $\left(\sim 1 \mathrm{~mm} \cdot \mathrm{h}^{-1}=360 \mathrm{~mm} \cdot \mathrm{s}^{-1}\right)$. By balancing the viscous drag and the buoyant forces it is possible to determine the viscosity required to achieve this velocity for a microbubble of a given diameter. For microbubbles up to $10 \mu \mathrm{m}$ diameter, a fluid with a viscosity of at least $0.12 \mathrm{Pas}$ would be necessary. At $2 \%$ concentration, a viscosity greater than this is found for shear rates less than $40 \mathrm{~s}^{-1}$ which suggests that the contrast agent will remain stable for a wide range of flow rates from rest to around $10 \mathrm{mms}^{-1}$ in sandstone-like samples.

The similarity of the diffusion of water within the gel structure to that of bulk water suggests that the contrast generated will be as high as possible without introducing any drifts due to the migration of the microbubbles.

Figure 4 can be used to determine the velocity of a given bubble diameter in either preparation. In methyl cellulose, the observed advection velocity of $8 \times 10^{-8} \mathrm{~m} \cdot \mathrm{s}^{-1}$ found using time lapse photography in methyl cellulose (Figure 6), should correspond to a bubble radius of $13 \mu \mathrm{m}$ which is within the size distribution observed in our preparations [13]. For these microbubbles, a velocity of $2 \times 10^{-13} \mathrm{~m} \cdot \mathrm{s}^{-1}$ is expected in gellan gum suggesting that no measurable movement should be seen in this experiment which is also in agreement with Figure 6.

The migration of the microbubbles is shown to be eliminated over the course of the MRI experiments as the vertical and horizontal profiles present no measurable gradient over time. The averaged signal intensity results demonstrate that the sensitivity is solely due to the presence of gas filled microbubbles as the de-gassed sample yields no measurable sensitivity. For experiments conducted at pressures below 1.4 bar, at which the compressibility of the lipid membrane becomes insufficient to cope with the tendency of the gas to dissolve in the surrounding medium, the signal intensity exhibits excellent correlation with the external pressure. Once this limit is exceeded and the fluid returned to atmospheric conditions, the microbubbles experience a change in size or rupture, which reduces the sensitivity. For several cycles of pressure, the sensitivity can be lost entirely.

\section{Conclusions}

A novel contrast agent, which allows stable MRI measurement of fluid pressure over time, has been produced using lipid coated microbubbles suspended in gellan gum. This has been achieved whilst maintaining a sensitivity as high as $38 \%$ signal change per bar.

The properties of the gellan gum have been assessed using rheology and diffusion NMR. Predicted and measured microbubble advection velocities have been compared using analytical methods and time lapse photography.

By combining the microbubbles with an equal amount of glycerol before their suspension in gellan gum, a preparation free of advective and amalgamative drifts has been used for bulk fluid pressure measurements over a period of at least 25 minutes.

Darcy's law (for Newtonian fluids) is the most relevant model for the majority of chemical engineering applications including oil recovery. Although the Gellan gum used in this study has many benefits for the imaging of pressure, its non Newtonian behaviour poses challenges for use in such applications.

However, the well characterized, small phase lag in the MR signal change (caused by the time required for water molecules to diffuse the lengthscale of the typical microbubble field gradient) following any pressure variation, offers the exciting possibility of imaging fast transients in pressure if the imaging gradient pulses are accurately synchronised.

Pressure gradients in a fluid flowing through a sandstone-like sample could be measured, provided that the width of the microbubble size distribution is suitably reduced.

The gellan gum base could also be used with microbubbles of different composition [22] to possibly measure greater pressure ranges. 


\section{Acknowledgments}

We gratefully acknowledge the EPSRC for support under grant number EP/C535219/1, Prof. Peter Morris (University of Nottingham) for facilitating access to an MRI Scanner and Dr. Peter Gardner (Royal Holloway Institute for Environmental Research) for supplying vacuum equipment.

\section{Appendix A.}

In this appendix a semi-analytical proof of Eq. 8 for the optimum echo time setting is provided.

Consider the signal from the homogeneous volume defined in Figure 1, which is given by the integral,

$$
A\left(T_{2}^{e f f}, T_{E}^{e f f}\right)=\int_{o}^{2 T_{E}^{e f f}}\left|\frac{2 \sin \left(\gamma G_{y} y_{o}\left(t-T_{E}^{e f f}\right)\right)}{\gamma G_{y}\left(t-T_{E}^{e f f}\right)}\right| e^{-t / T_{2}^{e f f}} d t
$$

By defining $\alpha=\gamma G_{y} y_{\mathrm{o}}$ and performing the substitution $x=\alpha\left(t-T_{E}^{e f f}\right)$, this integral can be simplified to,

$$
A\left(T_{2}^{e f f}, T_{E}^{e f f}\right)=\frac{2 y_{o}}{\alpha} F\left(\alpha T_{2}^{e f f}, \alpha T_{E}^{e f f}\right)
$$

where the function $F\left(\alpha T_{2}^{\text {eff }}, \alpha T_{E}^{\text {eff }}\right)$ has been defined as,

$$
F\left(\alpha T_{2}^{e f f}, \alpha T_{E}^{e f f}\right)=e^{-T_{E}^{e f f} / T_{2}^{e f f}} G\left(\alpha T_{2}^{e f f}, \alpha T_{E}^{e f f}\right)
$$

and the function $G\left(\alpha T_{2}^{\text {eff }}, \alpha T_{E}^{\text {eff }}\right)$ is given by the integral,

$$
G\left(\alpha T_{2}^{e f f}, \alpha T_{E}^{e f f}\right)=\int_{-\alpha T_{E}^{e f f}}^{\alpha T_{E}^{e f f}}|\operatorname{sinc}(x)| e^{\frac{-x}{\alpha T_{2}^{e f f}}} d x
$$

For the volume defined in Figure 1, the maximum measured frequency in the experiment is given by,

$$
F_{\max }=\frac{\not G_{y} y_{\max }}{2 \pi}
$$

To prevent Nyquist ghosts, the highest frequency that is measured must be at least twice the highest frequency originating from the NMR. Using $N b / T_{E}^{\text {eff }}$ as the sampling rate and setting the field of view to twice the extent of the sample results in,

$$
\frac{N b}{T_{E}^{e f f}}=\frac{\gamma G_{y} y_{o}}{\pi}
$$

where $N b$ is the number of digital points sampled (typically 70 or more). Assuming that $T_{2}^{\text {eff }}$ and $T_{E}^{\text {eff }}$ are in the order of $2 \mathrm{~s}$ and $0.5 \mathrm{~s}$ respectively, the expected value of $\alpha$ is typically in excess of 140 for which the exponential term in Eq. (A4) tends to 1 giving:

$$
G\left(\alpha, T_{2}^{e f f}, T_{E}^{e f f}\right) \approx \int_{-\alpha T_{E}^{e f f}}^{\alpha T_{E}^{e f f}} \operatorname{sinc}(x) \mid d x
$$

For sufficiently large values of $\alpha T_{E}$, the integral of the modulus of the $\operatorname{sinc}(x)$ term is independent of $T_{2}^{E f f}$ which gives

$$
F\left(\alpha T_{2}^{e f f}, \alpha T_{E}^{e f f}\right) \approx e^{-T_{E}^{e f f}} / T_{2}^{e f f}
$$

The maximum of the first derivative of $F$ with respect to $T_{2}^{\text {eff }}$ gives the optimum setting for $T_{E}$. This can be found by setting the second derivative to zero,

$$
\frac{d^{2} F}{d T_{2}^{e f f 2}}=\left(\frac{-2 T_{E}^{e f f}}{T_{2}^{e f f 3}}\right) F\left(1-\frac{T_{E}^{e f f}}{2 T_{2}^{e f f}}\right)=0
$$

which gives,

$$
T_{E}^{e f f}=2 T_{2}^{e f f}
$$

This semi-analytical solution for the optimum echo time agrees with the result found from the Monte Carlo simulation.

\section{References}

[1] M. Bencsik, And C. Ramanathan, Direct Measurement Of Porous Media Local Hydrodynamical Permeability Using Gas MRI. Magnetic Resonance Imaging 19 (2001) 379-383.

[2] R. Weisskoff, C. Zuo, J. Boxerman, And B. Rosen, Microscopic Susceptibility Variation And Transverse Relaxation - Theory And Experiment. Magnetic Resonance In Medicine 31 (1994) 601-610.

[3] A. Alexander, T. Mccreery, T. Barrette, A. Gmitro, And E. Unger, Microbubbles As Novel Pressure-Sensitive MR Contrast Agents. Magnetic Resonance In Medicine 35 (1996) 801-806.

[4] R. Dharmakumar, D. Plewes, And G. Wright, On The Parameters Affecting The Sensitivity Of Mr Measures Of Pressure With Microbubbles. Magnetic Resonance In Medicine 47 (2002) 264-273.

[5] R. Dharmakumar, D. Plewes, And G. Wright, A Novel Microbubble Construct For Intracardiac Or Intravascular Mr Manometry: A Theoretical Study. Physics In Medicine And Biology 50 (2005) 47454762.

[6] R. Morris, M. Bencsik, A. Vangala, And Y. Perrie, Three-Dimensional Fluid Pressure Mapping In Porous Media Using Magnetic Resonance Imaging With Gas-Filled Liposomes. Magnetic Resonance Imaging 25 (2007) 509-512.

[7] R. Glaser, And R.B. Glaser, Biophysics, Springer, New York, 2000.

[8] H.A. Barnes, J.F. Hutton, And K. Walters, An Introduction To Rheology, Elsevier, 1989.

[9] P. Rajitha, R. Chhabra, N. Sabiri, And J. Comiti, Drag On NonSpherical Particles In Power Law Non-Newtonian Media. International Journal Of Mineral Processing 78 (2006) 110-121.

[10] P.A.E. Williams, And G.O.E. Phillips, Gums And Stabilisers For The Food Industry 11, Royal Society Of Chemistry, 2002.

[11] J. Hennig, A. Nauerth, And H. Friedburg, Rare Imaging - A Fast Imaging Method For Clinical Mr. Magnetic Resonance In Medicine 3 (1986) 823-833

[12] E. Schutt, D. Klein, R. Mattrey, And J. Riess, Injectable Microbubbles As Contrast Agents For Diagnostic Ultrasound 
Imaging: The Key Role Of Perfluorochemicals. Angewandte ChemieInternational Edition 42 (2003) 3218-3235.

[13] A. Vangala, R. Morris, M. Bencsik, And Y. Perrie, Preparation And Characterization Of Gas-Filled Liposomes: Can They Improve Oil Recovery? J Liposome Res 17 (2007) 263-72.

[14] S. Cho, J. Kim, And J. Kim, Dynamic Surface Tension Of Stable AirFilled Microbubbles Prepared By Freeze-Drying A Solution Of Lipid/Surfactant Mixture. Colloids And Surfaces A-Physicochemical And Engineering Aspects 284 (2006) 453-457.

[15] A. Vangala, G. Kalkat, And Y. Perrie, Application Of Gas-Filled Liposomes As Pressure Probes For Oil Extraction: A Novel And Simple Preparation Technique. Journal Of Pharmacy And Pharmacology 58 (2006) A41-A41.

[16] N. Nestle, P. Galvosas, O. Geier, S. Vasenkow, And J. Kärger, PfgNMR Diffusion Studies In Polysaccharide Gels With And Without Inner Magnetic Field Gradients, 15th European Experimental NMR Conference (Eenc 2000), University Of Leipzig, 2000.

[17] M. Renaud, M. Belgacem, And M. Rinaudo, Rheological Behaviour Of Polysaccharide Aqueous Solutions. Polymer 46 (2005) 1234812358.

[18] Cpkelco, Kelcogel Gellan Gum Book, Atlanta, 2007.

[19] M. Mooney, And R.H. Ewart, The Conicylindrical Viscometer. Physics 5 (1934) 5.

[20] P. Galvosas, F. Stallmach, G. Seiffert, J. Karger, U. Kaess, G. Majer, And Zv, Generation And Application Of Ultra-High-Intensity Magnetic Field Gradient Pulses For NMR Spectroscopy. Journal Of Magnetic Resonance 151 (2001) 260-268.

[21] M. Holz, S.R. Heil, A. Sacco, And Hl, Temperature-Dependent SelfDiffusion Coefficients Of Water And Six Selected Molecular Liquids For Calibration In Accurate H-1 NMR Pfg Measurements. Physical Chemistry Chemical Physics 2 (2000) 4740-4742.

[22] F. Gerber, M. Krafft, G. Waton, And T. Vandamme, Microbubbles With Exceptionally Long Life - Synergy Between Shell And Internal Phase Components. New Journal Of Chemistry 30 (2006) 524-527. 